\title{
Multiscale Composites: Assessment of a Feasible Manufacturing Process
}

\author{
R. Volponi ${ }^{D},{ }^{1}$ P. Spena, ${ }^{1}$ F. De Nicola, ${ }^{1}$ and L. Guadagno ${ }^{2}$ \\ ${ }^{1}$ CIRA Italian Aerospace Research Centre, Prototypes Composite Materials Lab, Via Maiorise Capua, Italy \\ ${ }^{2}$ Department of Industrial Engineering-DIIn-Università di Salerno, Via Giovanni Paolo II, 132-84084 Fisciano, Italy \\ Correspondence should be addressed to R. Volponi; r.volponi@cira.it
}

Received 27 December 2018; Revised 25 February 2019; Accepted 4 April 2019; Published 13 May 2019

Guest Editor: Susanna Laurenzi

Copyright (C) 2019 R. Volponi et al. This is an open access article distributed under the Creative Commons Attribution License, which permits unrestricted use, distribution, and reproduction in any medium, provided the original work is properly cited.

\begin{abstract}
A very interesting field of research on advanced composite materials is the possibility to integrate new functionalities and specific improvements acting on the matrix of the composite by means of a nanocharged resin. In this way, the composite becomes a so-called "multiscale composite" in which the different phases change from nano to macro scale. For example, the incorporation of nanoscale conductive fillers with intrinsically high electrical conductivity could allow a tailoring of this property for the final material. The properties of carbon nanotubes (CNT) make them an effective candidate as fillers in polymer composite systems to obtain ultralight structural materials with advanced electrical and thermal characteristics. Nevertheless, several problems are related to the distribution in the matrix and to the processability of the systems filled with CNT. Existing liquid molding processes such as resin transfer molding (RTM) and vacuum-assisted resin transfer molding (VARTM) can be adapted to produce carbon fiber reinforced polymer (CFRP) impregnated with CNT nanofilled resins. Unfortunately, the loading of more than $0.3-0.5 \%$ of CNT can lead to high resin viscosities that are unacceptable for such kind of processes. In addition to the viscosity issues that are related to the high CNT content, a filtration effect of the nanofillers caused by the fibrous medium may also lead to inadequate final component quality. This work describes the development of an effective manufacturing process of a fiber-reinforced multiscale composite panel, with a tetra-functional epoxy matrix loaded with carbon nanotubes to increase its electrical properties and with GPOSS to increase its resistance to fire. A first approach has been attempted with a traditional liquid infusion process. As already anticipated, this technique has shown considerable difficulties related both to the low level of impregnation achieved, due to the high viscosity of the resin, and to the filtration effects of the dispersed nanocharges. To overcome these problems, an opportunely modified process based on a sort of film infusion has been proposed. This modification has given an acceptable result in terms of impregnation and morphological arrangement of CNTs in nanofilled CFRP. Finally, the developed infiltration technique has been tested for the manufacture of a carbon fiber-reinforced panel with a more complex shape.
\end{abstract}

\section{Introduction}

The inclusion of nanomaterials inside a polymer allows us to add new functional characteristics that increase the range of possible uses of the polymer [1].

Clearly, the inclusion of nanofillers in a polymer does not highly affect the final mechanical properties of the composite $[2,3]$. Nevertheless, it is possible to take full advantage of the additional features provided by the nanocharge using them as matrices in fiber reinforced composites. Indeed, this approach can give rise to composite systems that show additional properties that derive from synergic effects by the coupling of different materials.

Starting from the combination of different materials with different scales of size (nano, micro, and macro), it is possible to develop a new class of advanced composite materials, commonly referred to as multiscale composites [4], which implement new intrinsic functionalities both in the purely structural and in the non-structural environment.

Concerning the mechanical performance, for example, it is possible to improve the adhesion between the layers of a composite in order to reduce delamination [5] or to improve 


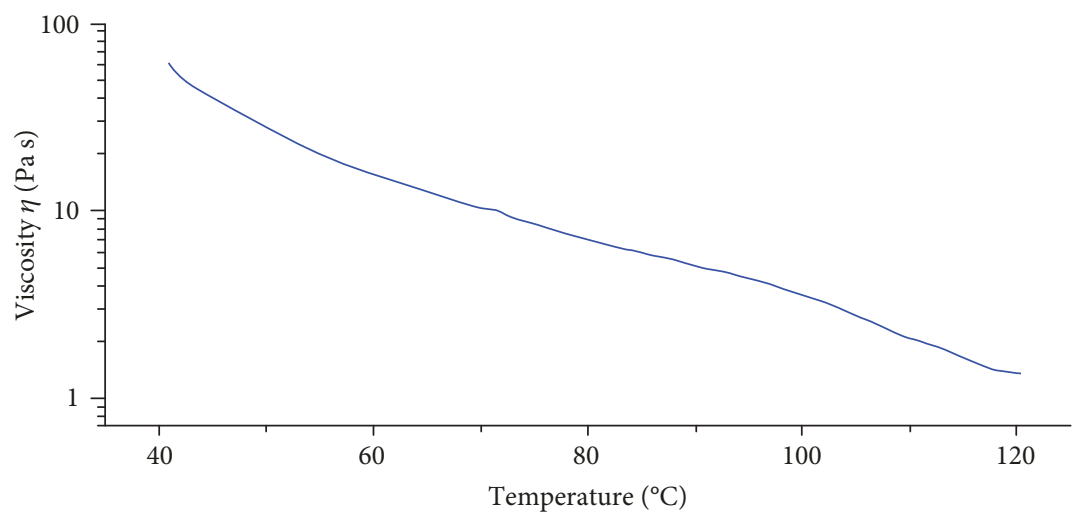

(a)

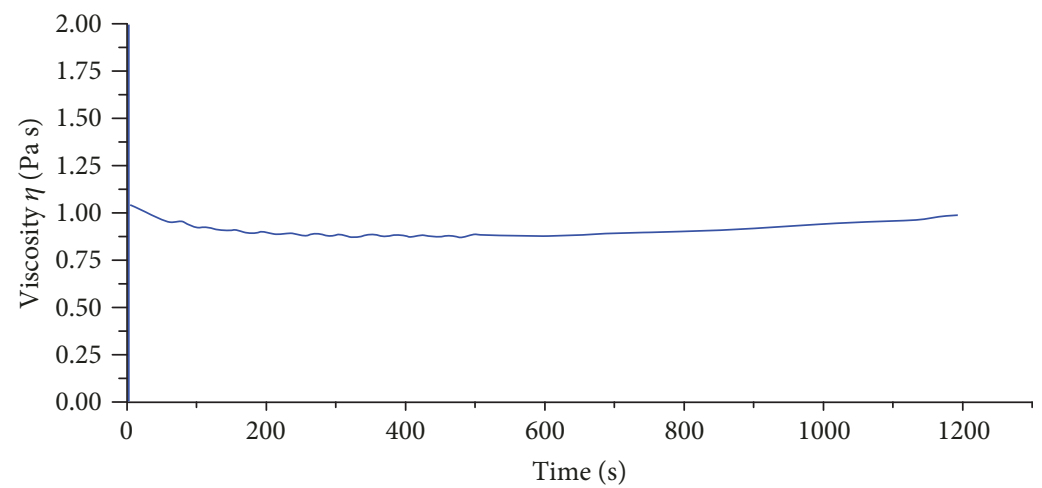

(b)

Figure 1: (a) Viscosity vs. temperature and (b) evaluation of the resin's pot life.

impact resistance or acoustic damping [6]. Furthermore, it is possible to develop features that make these materials "intelligent" as self-repairing materials [7] or sensitive to any damage suffered [8] or with a reduced radar section [9].

In a manufacturing process of a multiscale composite, it is possible to distinguish two basic phases:

(i) The inclusion of nanocharges (either in the matrix or on the fiber surface) [10]

(ii) Impregnation of reinforcing fibers with the matrix

A practicable approach for the realization of a multiscale composite can be firstly to disperse the nanometric charge in the polymer, thus creating a nanocomposite, and subsequently use this nanocomposite to impregnate the reinforcing fibers.

An alternative approach for the realization of a multiscale composite consists initially in distributing the nanoparticles on the surface of the fibers by means of a spray, and using a chemical graft that acts as a bridge. Other methods to disperse the nanocharges use electrophoresis or even growth of nanotubes directly on the fibers and then proceed with the infusion of the pure matrix [11].

The dispersion of nanocharges in a polymer is the most common method for the inclusion of nanomaterials into a multiscale composite. Nanomaterials, especially carbon nanofibers or nanotubes, have a strong tendency to agglomerate. Therefore, the purpose of the dispersion process is to break the agglomerates in order to keep the individual nanomaterials separated and to distribute them uniformly inside the polymer [12]. The difficulty of the dispersion process increases with the growth of the relative surfaces of the nanostructure defined as aspect ratio. In fact, the forces that tend to aggregate the various nanostructures are Van der Waals forces and become very high due to the large contact surface that occurs between small particles [13]. An immediate effect of such behavior is the sudden rising of the viscosity of the polymer during the dispersion [14-16].

In order to define a feasible CFRP manufacturing process, it is fundamental to study the rheological characteristics of the multifunctionalized resins proposed. From this study, it is possible to evaluate the viscosity and the time available for the manufacture of the composite (pot life).

Based on the evaluation of these parameters, it is possible to propose a method to produce the composite.

\section{Experiments}

2.1. Materials. The epoxy system used in this activity is based on a mixture of tetraglycidyl methylene dianiline (TGMDA) and 1,4-butandiol diglycidy lether (BDE) in which $0.5 \%$ of carbon nanotubes and 5\% of GPOSS [16] were dispersed.

The evaluation of the viscosity of the nanofilled resin has been made using a rheometer TA Discovery HR-2 at different temperatures in a first test (Figure 1(a)) and holding the sample at $120^{\circ} \mathrm{C}$, to estimate the pot life of the system. 


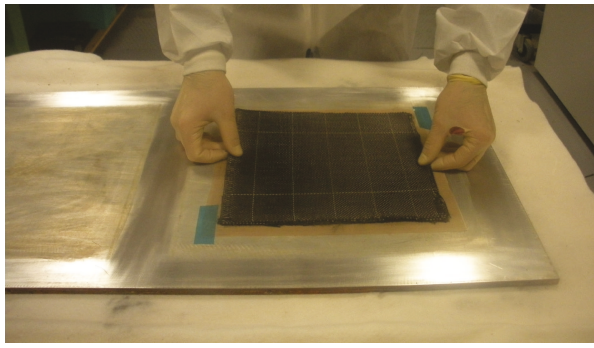

(a)

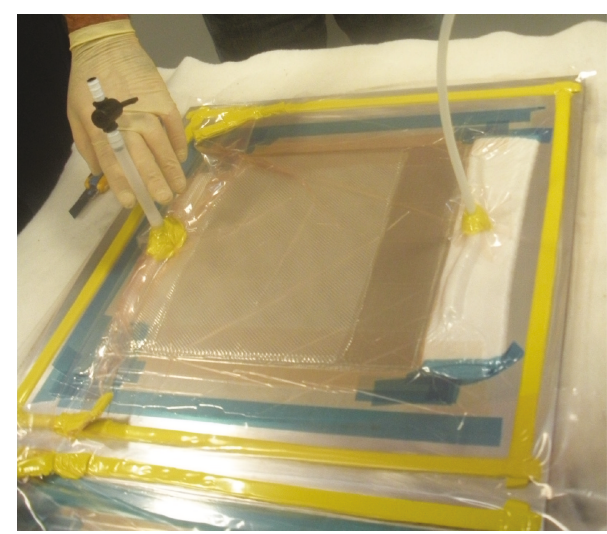

(b)

FIgURe 2: (a) Placing of the dry preform and (b) vacuum bag.

It is possible to see that the value of viscosity at $120^{\circ} \mathrm{C}$ is about 1.0 Pas and keeps constant at this level for at least 20 minutes (Figure 1(b)). We anticipate that this value is very close to the threshold level that it is possible to find in literature as the practical limit of the infusion process [17].

2.2. Classical Infusion Scheme for a Flat Panel. Based on this viscosity behavior, a manufacturing test has been done on a flat panel, 7 plies [0/90], adopting a classical infusion scheme.

The fiber fabric used for the test article is Toho Tenax G30550 3K HTA 5131 200tex/PW (alternatively indicated as HTA40 E13 3K 200tex Aero/TR/1067 mm). This fabric is especially designed to facilitate the permeation of resin.

The mold plate was coated with a water-based PTFE release agent and covered by one layer of nonporous release film. On the release film, 7 plies of carbon fiber fabric are layered (Figure 2(a)).

The laminate is covered by a porous release film, namely, a PTFE-coated fiberglass that allows the excess of resin, the volatiles, and the trapped air to flow into the breather during the cure process and by a layer of distribution medium covering almost the whole panel. A breather cloth is placed in correspondence to the outlet of the resin, and finally a vacuum bag is prepared (Figure 2(b)).

The panel on the mold and the resin reservoirs are thermostated at $120^{\circ}$ in the oven and then the infusion started taking 35 minutes to fill the dry preform (Figure 3).

Once the infusion was completed, the cure cycle started.

The panel was cured according to the following cure cycle: a ramp of $3^{\circ} \mathrm{C} / \mathrm{min}$ up to $190^{\circ} \mathrm{C}$, a hold for 3 hours, and a cooling phase with a rate of $2^{\circ} \mathrm{C} / \mathrm{min}$.

The panel shows many defects such as the low level of compactness and dry regions on the upper side (Figure 4(a)) and in the final part. The resin did not reach the lower side of the laminate where only small areas were infiltrated (Figure 4(b)).

Successively, an investigation based on microscope inspections was conducted on samples extracted from the panel.

From the microscope observation, it is possible to see the presence of a large number of voids, in particular in the regions far from the inlet of the resin (Figure 5).

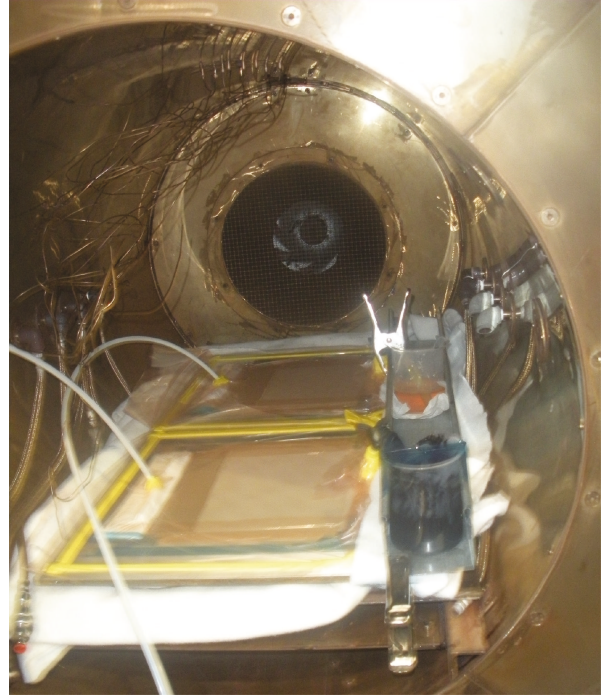

Figure 3: Infusion setup.

The experiment demonstrated the practical unfeasibility of the classical liquid infusion approach with the resin available. Indeed, despite the limited surface of the panel, the impregnation was not complete. This fact confirms the limit value of 0.8 Pas reported in literature for the resin viscosity in a liquid infusion process [17].

2.3. Modified Film Infusion Scheme for a Flat Panel. The solution of the critical point found during the classical infusion process of a simple flat panel has been attempted by means of a variation of resin film infusion (RFI) consisting in the infusion of a bulk of nanocharged resin spread on a release film. Successively, a dry preform $(400 \mathrm{~mm} \times 400 \mathrm{~mm})$ with 7 plies of carbon fiber fabric has been placed on this resin thick film (Figure 6(a)). The edges of the preform have been sealed to force the resin to flow through the thickness (Figure 6(b)).

The upper side of the laminate has been covered by a porous release film and by a distribution medium in order to allow the resin to flow on the surface. Around the preform, 


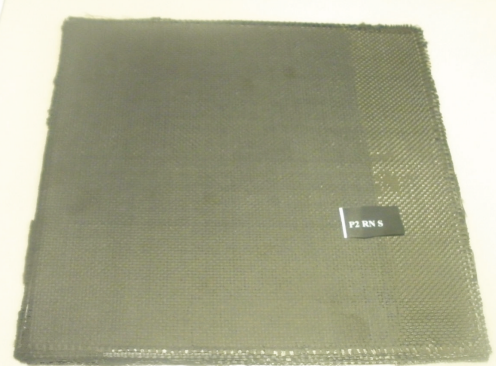

(a)

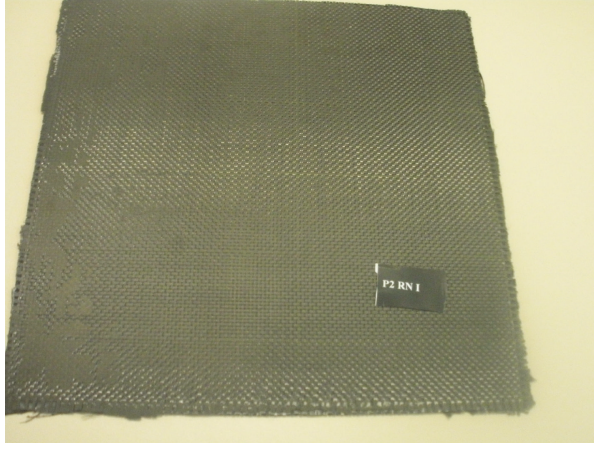

(b)

Figure 4: Final panel: (a) upper side and (b) lower side.
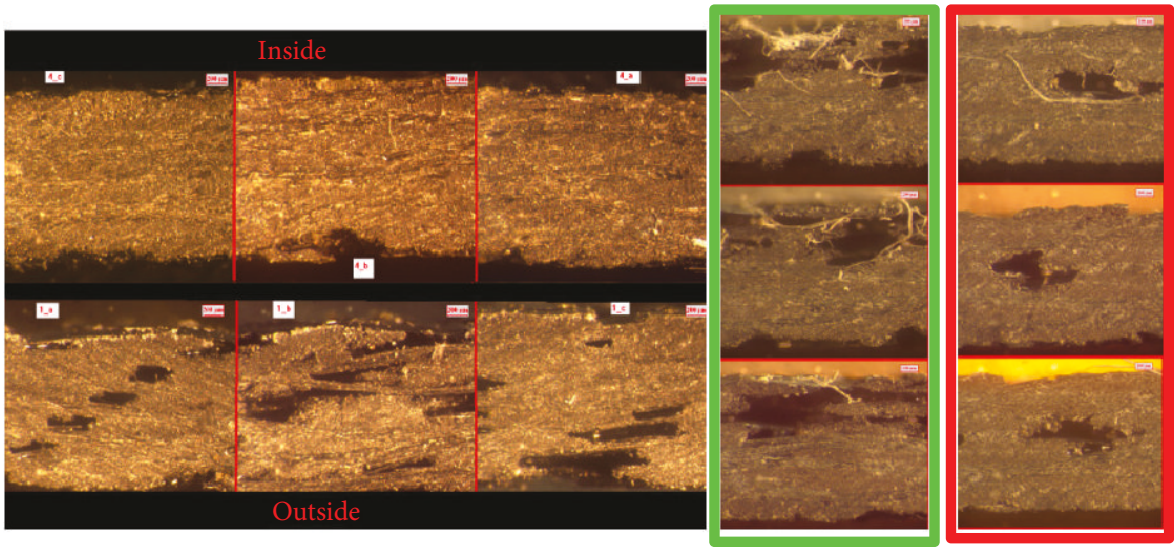

FIGURE 5: Inspection with an optical microscope.

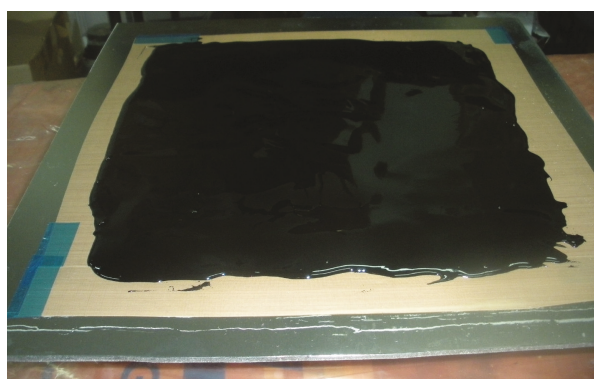

(a)

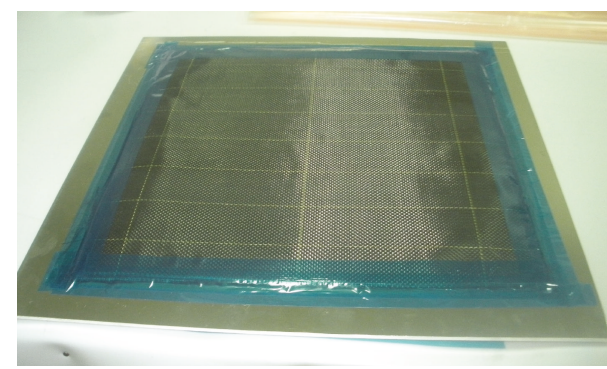

(b)

Figure 6: (a) Resin thick film distribution and (b) preform on the thick resin film.

a bleeder medium has been placed to receive the excess of resin, and a vacuum bag has been prepared (Figure $7(a)$ ). The laminate has been placed into the autoclave (Figure 7(b)).

The curing cycle has been set with the following parameters:

(i) First ramp to $120^{\circ} \mathrm{C}$ at $2^{\circ} \mathrm{C} / \mathrm{min}$, vacuum active

(ii) Dwell of 30 minutes at $120^{\circ} \mathrm{C}$

(iii) A second ramp to $180^{\circ} \mathrm{C}$ at $2^{\circ} \mathrm{C} / \mathrm{min}$

(iv) Dwell of three hours at $180^{\circ} \mathrm{C}$

\section{(v) Pressure at 7 bar, vent open}

The excess of resin has been absorbed by the bleeder/breather (Figure 8(a)). The panel is $1.39 \mathrm{~mm}$ thick, with a volume fiber fraction (calculated) of $V_{\mathrm{f}}=0.55$. The panel has been trimmed, and the final dimensions are $330 \times 330 \mathrm{~mm}$ (Figure 8(b)).

The quality of manufacturing has been verified cutting an edge of panel and observing it by means of an optical microscope at different magnitudes. The microscope analysis (Figure 9) shows that the panel does not have macroscopic defects and is uniformly infiltrated. Therefore, the feasibility 


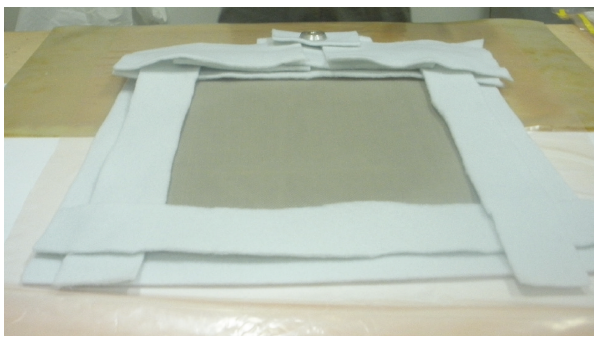

(a)

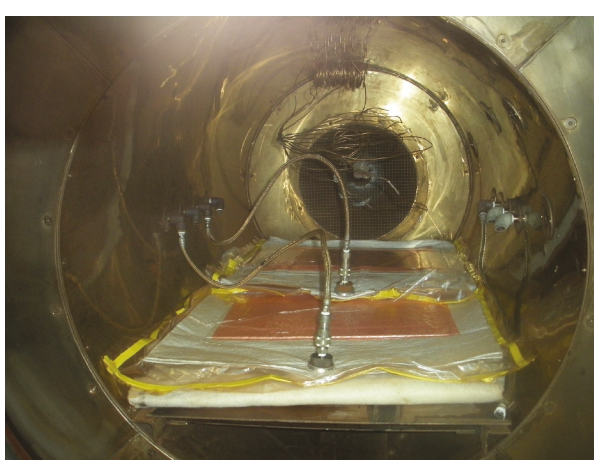

(b)

Figure 7: (a) Breather/bleeder around the preform and (b) laminated panel in the autoclave.

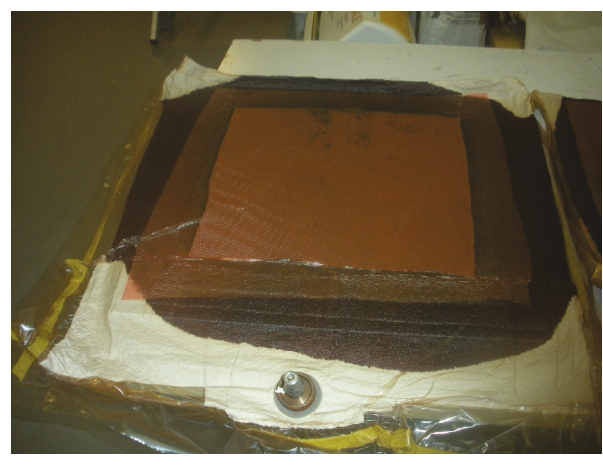

(a)

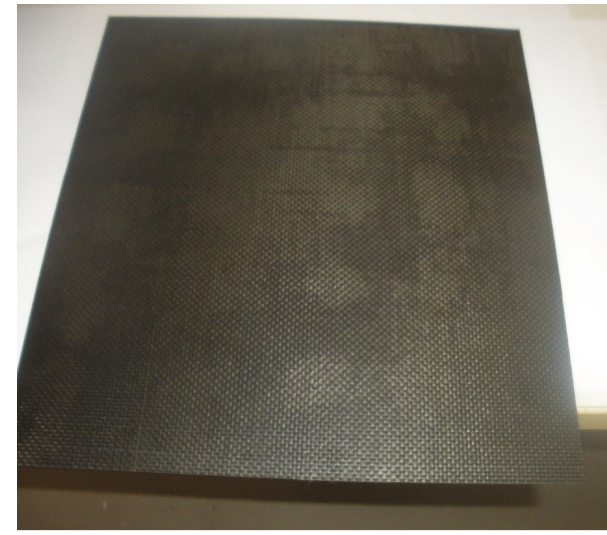

(b)

FIGURE 8: (a) Squeezing of the resin and (b) final result.

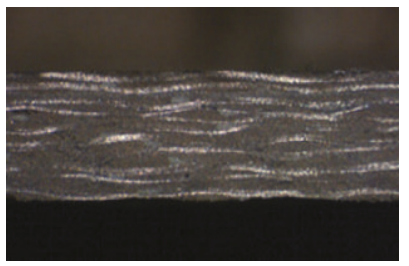

$25 \times$

(a)

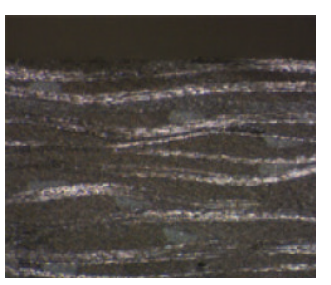

$150 \times$

(b)

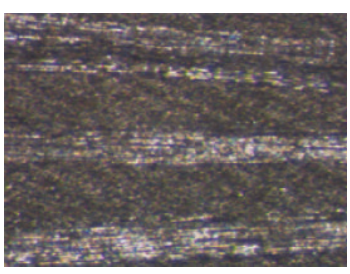

$350 \times$

(c)

FIgURE 9: Inspection with an optical microscope.

and effectiveness of this modified manufacturing technique have been proven for the realization of fiber-reinforced panels with nanocharged matrix.

2.4. Modified Film Infusion for a Panel with Stringers. Based on the foregoing positive results, the manufacturing of a more complex panel with three $\mathrm{T}$ stringers has been approached.

A rubber tool (Figure 10(a)) has been casted to allow for the lamination of the stringers and the compaction of the panel under the vacuum bag. The silicon rubber used in this case is Aircast 3700, by Airtech.

The rubber tool is placed on an external aluminium rigid plate which forces the flexible tool to stay in the correct position. The thermal expansion of this tool helps the compaction of the preform during the cure cycle.

The three $\mathrm{T}$ stringers have been prelaminated in the rubber tool to achieve the proper shape and successively extracted from the tool (Figure 10(b)). 


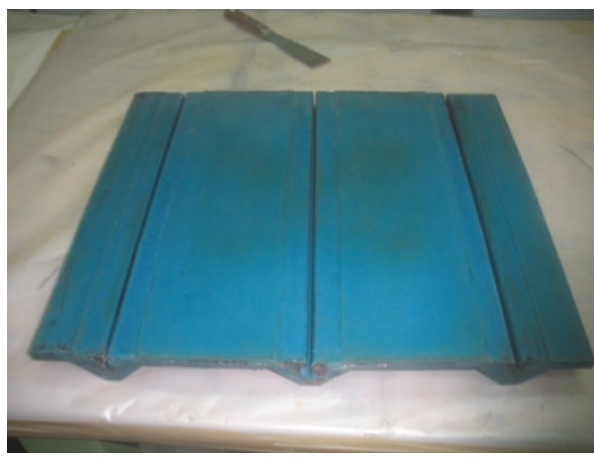

(a)

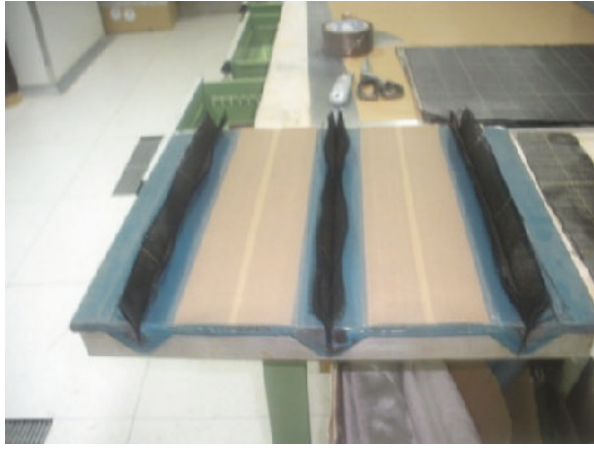

(b)

Figure 10: (a) Rubber tool and (b) T stringer preforming.

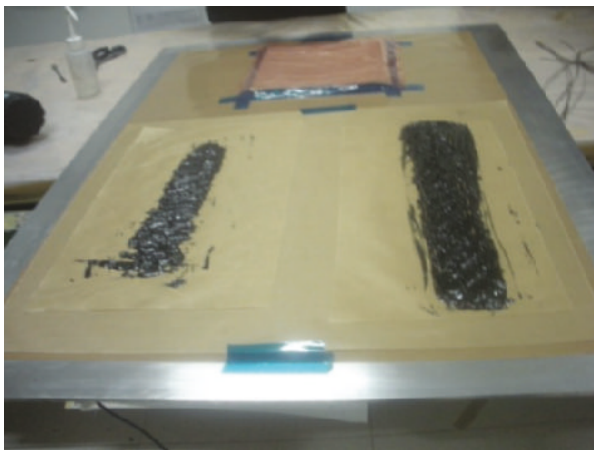

(a)

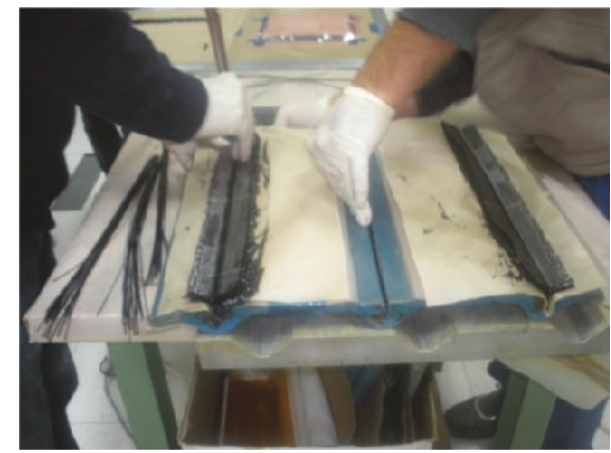

(b)

Figure 11: (a) Film resin for the T stringer and (b) deposition of the impregnated $\mathrm{T}$ stringer.

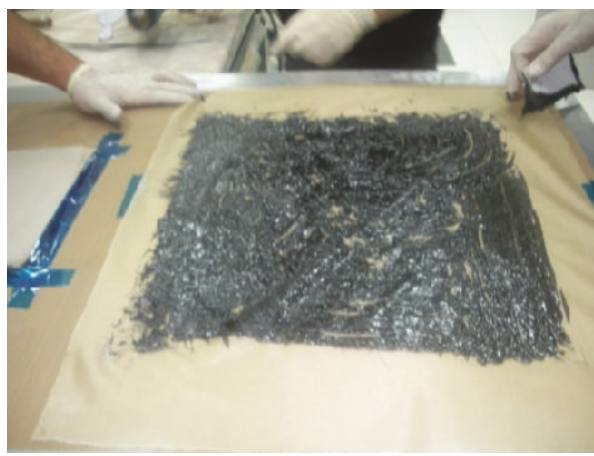

(a)

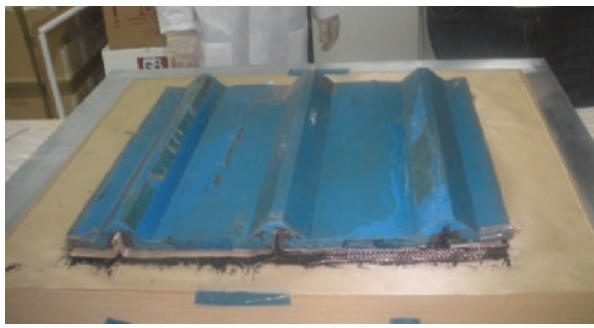

(b)

FIGURE 12: (a) Film of resin and (b) placing of the rubber tool.

The right quantity of resin for each stringer has been placed on separate stripes of porous peel ply (Figure 11(a)).

This is done to facilitate the insertion of resin and preforms in the cavities, by hand layup, in a very short time. The stripes and the preforms are placed in the cavity (Figure 11(b)).

The change in temperature causes a very rapid increase in viscosity which makes very difficult any other operation to disperse the resin on the surface.

On the metal mold, a large porous peel ply has been prepared with the right quantity of resin. The resin has been dispersed at the same height thanks to a specific tool
(Figure 12(a)), then the flat preform of the skin has been placed on the resin and finally the rubber tool, already prepared with stringer preforms, has been placed in the reverse position (Figure 12(b)).

The rigid metal outer mold has been placed on the rubber (Figure 13(a)), and a typical vacuum bag has been prepared. The overall assembly has been cured in the autoclave (Figure 13(b)). After the cure, the quantity of resin squeezed out from the panel was very limited.

The external face of the stringered panel appears very uniform. No major excess resin pocket regions are visible, 


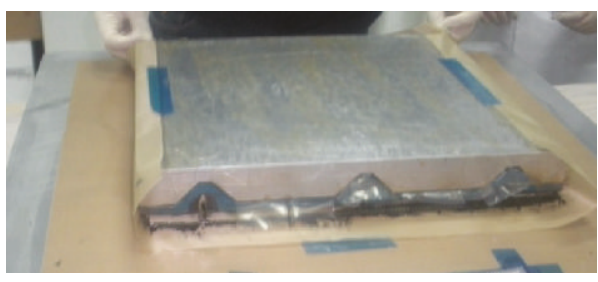

(a)

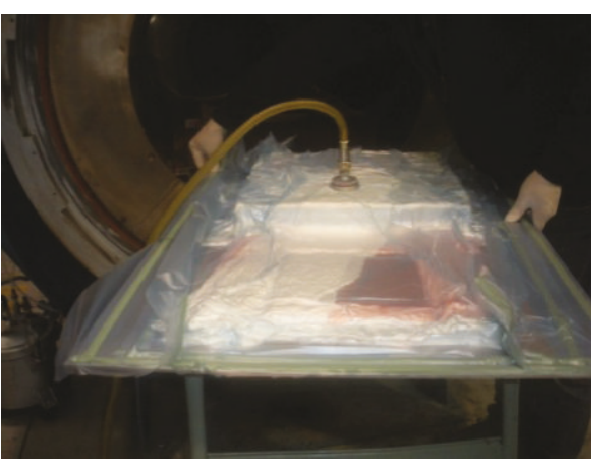

(b)

Figure 13: (a) Placing of a rigid metal outer mold and (b) vacuum bag and cure in the autoclave.

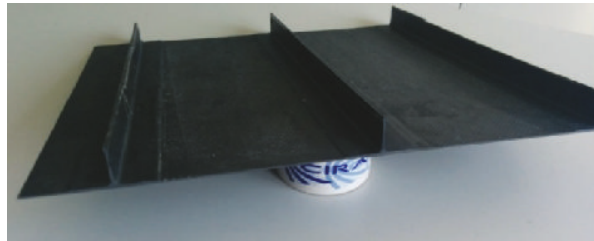

(a)

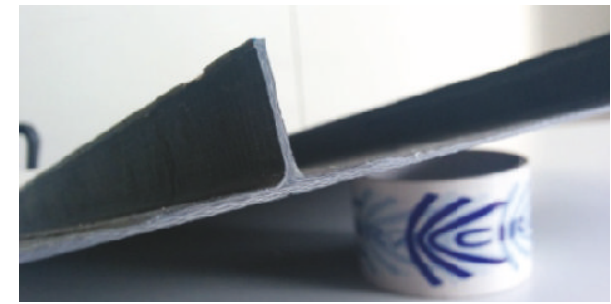

(b)

Figure 14: (a) Final panel with 3 co-cured $\mathrm{T}$ stringer and (b) $\mathrm{T}$ stringer particular.

except for the internal face of the panel where it is possible to notice small zones not perfectly uniform. However, the thickness appears quite homogeneous (Figure 14(a)), and there are no voids at the bottom of the T stringer (Figure 14(b)).

\section{Conclusion}

The experience described in this work shows the results obtained during the process of a multiscale composite material by means of nanocharged matrix, highlighting the difficulties incurred during the manufacturing of just a simple flat panel using a classical infusion process. In order to overcome manufacturing criticalities, a modified infusion scheme has been conceived and applied to a simple panel and to a stringered panel. Overall, the possibility to produce stringered complex panels with nanofilled resin has been demonstrated using the described manufacturing approach, even though at this preliminary stage some imperfections are still present. These imperfections are due to the very strict conditions in which it is possible to operate with the filled resin to distribute it manually on large surfaces. An option to be tested is to heat up the tool and all the materials involved at the same temperature, even though it is difficult to operate manually. In this case, an automated uniform distribution of resin at high temperature on the hot tool could be a viable option to ameliorate the result.

The final aim of this work is to demonstrate the potentiality and the process feasibility of these materials to be integrated in composite structures with additional features.

\section{Data Availability}

The data used to support the findings of this study are available from the corresponding author upon request.

\section{Conflicts of Interest}

The authors declare that they have no conflicts of interest.

\section{Acknowledgments}

The research leading to these results has received funding from the European Union's Seventh Framework Programme for research, technological development, and demonstration under Grant Agreement N 313978.

\section{References}

[1] B. Fiedler, F. H. Gojny, M. H. G. Wichmann, M. C. M. Nolte, and K. Schulte, "Fundamental aspects of nano-reinforced composites," Composites Science and Technology, vol. 66, no. 16, pp. 3115-3125, 2006.

[2] M. C. L. McCrary-Dennis and O. I. Okoli, "A review of multiscale composite manufacturing and challenges," Journal of Reinforced Plastics and Composites, vol. 31, no. 24, pp. 16871711, 2012.

[3] G. Barra, F. De Nicola, B. De Vivo et al., "Enhanced electrical properties of carbon fiber reinforced composites obtained by an effective infusion process," in 2014 IEEE 9th Nanotechnology Materials and Devices Conference (NMDC), Aci Castello, Italy, October 2014. 
[4] E. Bekyarova, E. T. Thostenson, A. Yu et al., "Multiscale carbon nanotube-carbon fiber reinforcement for advanced epoxy composites," Langmuir, vol. 23, no. 7, pp. 3970-3974, 2007.

[5] D. Lefevre, S. Comas-Cardona, C. Binetruy, and P. Krawczak, "Coupling filtration and flow during liquid composite molding: experimental investigation and simulation," Composites Science and Technology, vol. 69, no. 13, pp. 2127-2134, 2009.

[6] A. Godara, L. Mezzo, F. Luizi et al., "Influence of carbon nanotube reinforcement on the processing and the mechanical behaviour of carbon fiber/epoxy composites," Carbon, vol. 47, no. 12, pp. 2914-2923, 2009.

[7] S. U. Khan, C. Y. Li, N. A. Siddiqui, and J.-K. Kim, "Vibration damping characteristics of carbon fiber-reinforced composites containing multi-walled carbon nanotubes," Composites Science and Technology, vol. 71, no. 12, pp. 1486-1494, 2011.

[8] M. Raimondo, F. De Nicola, R. Volponi et al., "Self-repairing CFRPs targeted towards structural aerospace applications," International Journal of Structural Integrity, vol. 7, no. 5, pp. 656-670, 2016.

[9] R. Volponi, F. de Nicola, and P. Spena, "Nanocomposites for new functionalities in multiscale composites," MATEC Web of Conferences, vol. 188, article 01027, 2018.

[10] D. Micheli, C. Apollo, R. Pastore, R. Bueno Morles, S. Laurenzi, and M. Marchetti, "Nanostructured composite materials for electromagnetic interference shielding applications," Acta Astronautica, vol. 69, no. 9-10, pp. 747-757, 2011.

[11] K. H. Hung, W. S. Kuo, T. H. Ko, S. S. Tzeng, and C. F. Yan, "Processing and tensile characterization of composites composed of carbon nanotube-grown carbon fibers," Composites Part A: Applied Science and Manufacturing, vol. 40, no. 8, pp. 1299-1304, 2009.

[12] F. Zaccardi, M. G. Santonicola, and S. Laurenzi, "Quantitative assessment of nanofiller dispersion based on grayscale image analysis: a case study on epoxy/carbon nanocomposites," Composites Part A: Applied Science and Manufacturing, vol. 115, pp. 302-310, 2018.

[13] A. Jiménez-Suárez, M. Campo, M. Sánchez, C. Romón, and A. Ureña, "Dispersion of carbon nanofibres in a low viscosity resin by calendering process to manufacture multiscale composites by VARIM," Composites Part B: Engineering, vol. 43, no. 8, pp. 3104-3113, 2012.

[14] E. F. Reia da Costa, A. A. Skordos, I. K. Partridge, and A. Rezai, "RTM processing and electrical performance of carbon nanotube modified epoxy/fibre composites," Composites Part A: Applied Science and Manufacturing, vol. 43, no. 4, pp. 593602,2012

[15] B.-C. Wang, X. Zhou, and K.-M. Ma, "Fabrication and properties of CNTs/carbon fabric hybrid multiscale composites processed via resin transfer molding technique," Composites Part B: Engineering, vol. 46, pp. 123-129, 2013.

[16] L. Guadagno, M. Raimondo, U. Vietri et al., "Effective formulation and processing of nanofilled carbon fiber reinforced composites," RSC Advances, vol. 5, no. 8, pp. 6033-6042, 2015.

[17] D. W. Becker, Tooling for Resin Transfer Moulding, Wichita State University, Wichita, Kansas. 


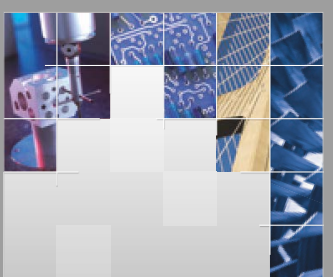

\section{Enfincering}
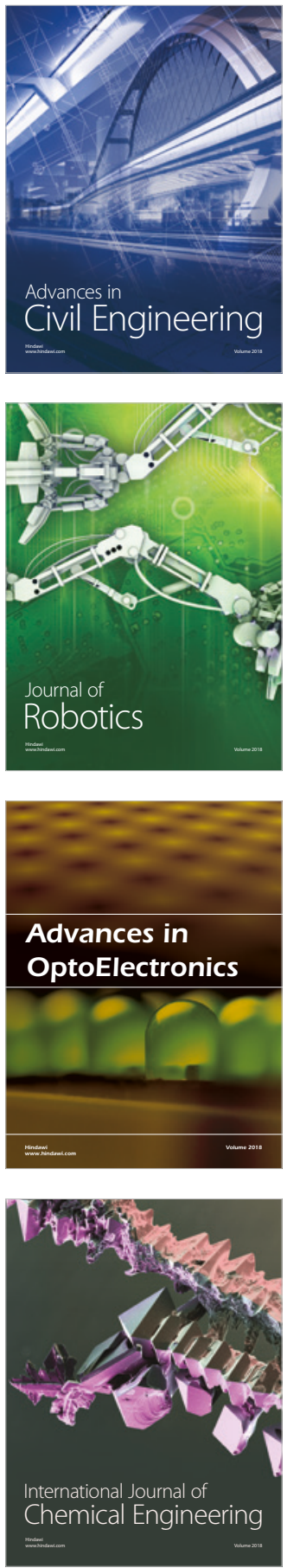

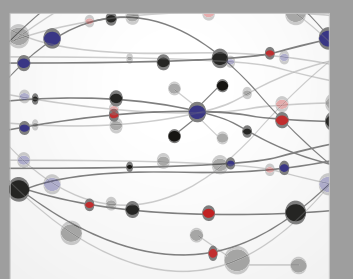

\section{Rotating \\ Machinery}

The Scientific World Journal

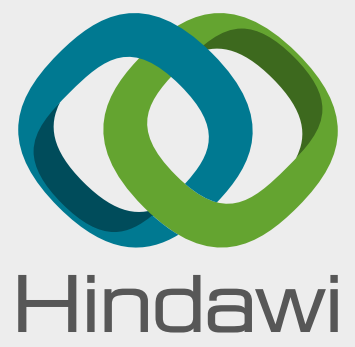

Submit your manuscripts at

www.hindawi.com
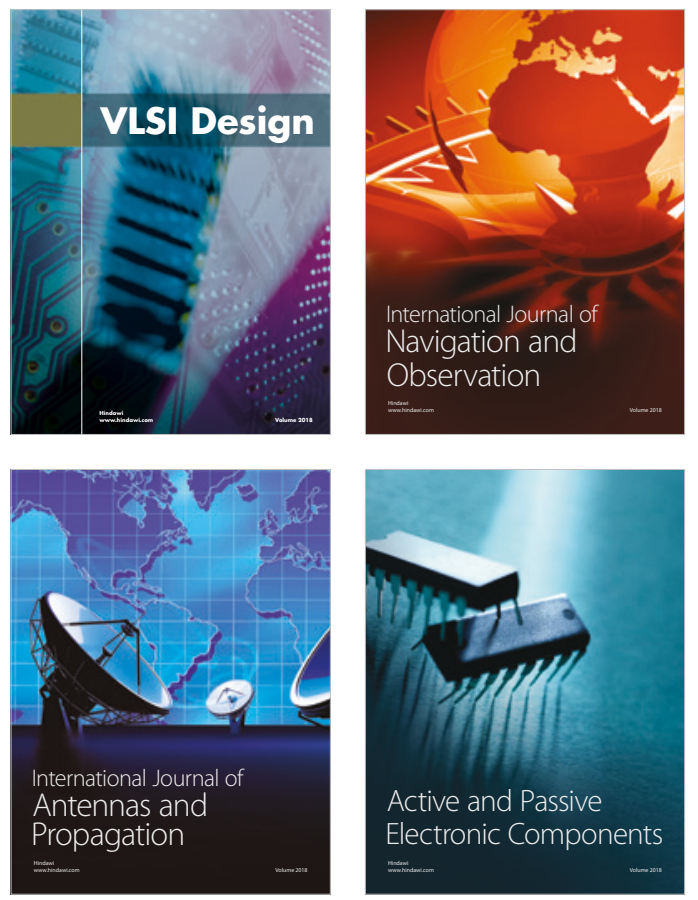
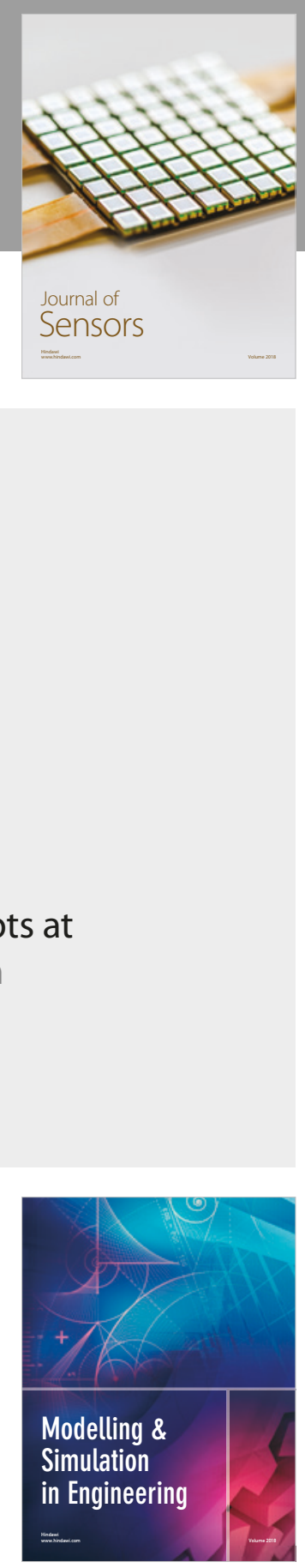

\section{Advances \\ Multimedia}
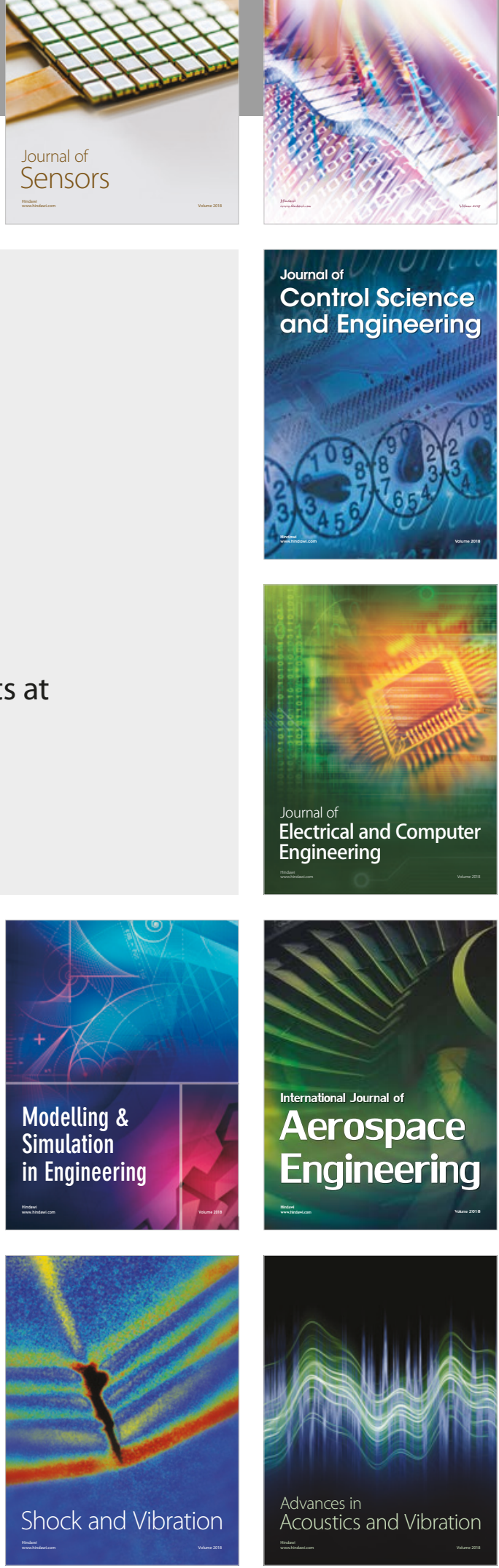